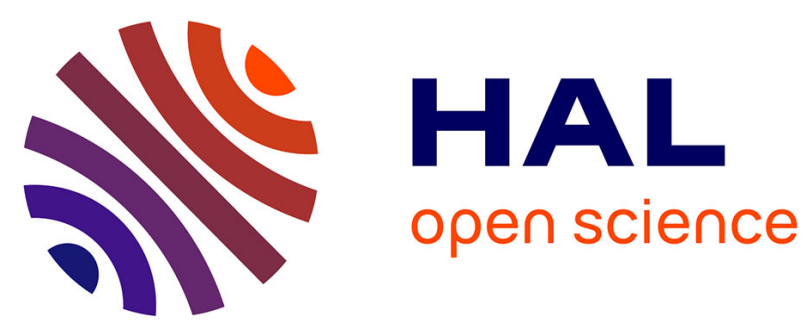

\title{
Simultaneous pressure-volume measurements using optical sensors and MRI 1 for left ventricle function assessment during animal experiment 2
}

Dima Abi-Abdallah Rodriguez, Emmanuel Durand, Ludovic de Rochefort, Younes Boudjemline, Elie Mousseaux

\section{To cite this version:}

Dima Abi-Abdallah Rodriguez, Emmanuel Durand, Ludovic de Rochefort, Younes Boudjemline, Elie Mousseaux. Simultaneous pressure-volume measurements using optical sensors and MRI 1 for left ventricle function assessment during animal experiment 2. Medical Engineering \& Physics, 2015, 37 (1), pp.100-108. hal-01123539

\section{HAL Id: hal-01123539 \\ https://hal.science/hal-01123539}

Submitted on 5 Mar 2015

HAL is a multi-disciplinary open access archive for the deposit and dissemination of scientific research documents, whether they are published or not. The documents may come from teaching and research institutions in France or abroad, or from public or private research centers.
L'archive ouverte pluridisciplinaire $\mathbf{H A L}$, est destinée au dépôt et à la diffusion de documents scientifiques de niveau recherche, publiés ou non, émanant des établissements d'enseignement et de recherche français ou étrangers, des laboratoires publics ou privés. 


\title{
Simultaneous pressure-volume measurements using optical sensors and MRI for left ventricle function assessment during animal experiment
}

\author{
Dima Abi-Abdallah Rodriguez ${ }^{a, b *}$, Emmanuel Durand ${ }^{a, b}$, Ludovic de Rocheforta ${ }^{a, b}$, Younes Boudjemline ${ }^{c, d}$ \\ and Elie Mousseaux ${ }^{\mathrm{c}, \mathrm{e}}$ \\ a Univ Paris-Sud, IR4M, UMR8081, Orsay, France \\ ${ }^{b}$ CNRS, IR4M, UMR8081, Orsay, France \\ ${ }^{c}$ Univ Paris 05, France \\ ${ }^{d}$ Hôpital Necker-Enfants malades, AP HP, Paris, France \\ eINSERM, U678, Paris, France
}

*Corresponding author email : dima.rodriguez@u-psud.fr

Word count : 4761 (excluding title, authors, tables/figures, figures legends and references)

\section{Abstract}

Simultaneous pressure and volume measurements enable the extraction of valuable parameters for left ventricle function assessment. Cardiac MR has proven to be the most accurate method for volume estimation. Nonetheless, measuring pressure simultaneously during MRI acquisitions remains a challenge given the magnetic nature of the widely used pressure transducers. In this study we show the feasibility of simultaneous in vivo pressure-volume acquisitions with MRI using optical pressure sensors. Pressure-volume loops were calculated while inducing three inotropic states in a sheep and functional indices were extracted, using single beat loops, to characterize systolic and diastolic performance. Functional indices evolved as expected in response to positive inotropic stimuli. The end-systolic elastance, representing the contractility index, the diastolic myocardium compliance, and the cardiac work efficiency all increased when inducing inotropic state enhancement. The association of MRI and optical pressure sensors within the left ventricle successfully enabled pressure-volume loop analysis after having respective data simultaneously recorded during the experimentation without the need to move the animal between each inotropic state. 


\section{Introduction}

The evaluation of the left ventricle (LV) performance is of high importance in physiologic investigation and clinical practice. The diastolic LV function can be assessed by evaluating relaxation properties and myocardium stiffness during the filling process. As for the systolic LV performance, it is governed by three principal factors, preload, afterload and the contractile or inotropic state of the myocardium. The first two determinants can be relatively easily measured or evaluated, but assessing contractile state is far more difficult. Any index intended to reflect contractility must be load-independent.

Common studies evaluate contractility using left ventricular stroke volume (SV), ejection fraction (EF) and cardiac output (CO). Though intuitive and simple, these parameters are load-dependent and consequently represent poor contractility indices. For instance, they are not good indicators of heart failure and cardiac dysfunction Maeder and Kaye [26], Gaddam and Oparil [11]. Other studies rely on pressure measurements alone to assess the LV performance. They evaluate, for example, the maximum rate of pressure change $\left(d P / d t_{\max }\right)$ which is known to be sensitive to inotropic state and thus correlates with cardiac contractility. Nonetheless, its load-dependence Grossman et al. [15], Kass et al. [19], Mason et al. [27] makes it a weak contractility indicator. The peak decline of pressure $\left(d P / d t_{\min }\right)$, on the other hand, can quantify isovolumic relaxation, but it cannot be qualified as an intrinsic relaxation index since it is preload-dependent Cohn et al. [6] as well as afterload-dependent Weiss et al. [47]. A widely used parameter for the relaxation quantification is the time constant $\tau$ which characterizes the pressure decay during isovolumic relaxation. Despite being preload-independent Varma et al. [46], $\tau$ cannot be considered an ideal index because of its afterload dependence.

No perfect assessment index can be obtained from volume or pressure alone. Indeed, volume or pressure measurements on their own are not sufficient to characterize the systolic performance, they cannot solely define contractility and cardiac response to inotropic agents. Simultaneous pressure and volume measurements are necessary to provide valuable functional parameters. Pressure indices can thus be coupled with volume information to negate load dependence. For example, rather than considering $d P / d t_{\max }$, the relationship of this value to end diastolic volume (EDV) can be computed by varying preload conditions. This relationship is linear and its slope provides a preload-independent contractility index Little [24]. The relaxation constant $\tau$ can also be evaluated over a range of afterloads and plotted against EDV Zile and 


\section{Brutsaert [49].}

In addition, a wide variety of indices that can be quantified by analyzing pressure-volume (PV) loops have been proposed to characterize the left ventricle systolic and diastolic performance. For instance a straightforward characterization of the myocardium stiffness can be obtained from the slope of the pressurevolume relationship during diastole. Furthermore, a measurement of the ventricular elastance when the contractile forces in the ventricle are at their peak, constitutes a good indicator of the ventricular contractility and systolic function. Known as end-systolic elastance $E_{e s}$, it is independent of preload, afterload and heart rate Brinke et al. [3], Suga [40]. The arterial system, as afterload, can also be assessed from the PV loop, and, like the ventricle chamber, it can be characterized by its elastance $E_{a}$. Studies have shown the importance of $E_{a}$ as a descriptor of the vascular load and its impact on cardiac performance Hayashida et al. [16], Kelly et al. [20], indicating that the ratio $E_{a} / E_{e s}$ quantifies the coupling between the ventricle and arterial system and governs ventriculoarterial matching. Additionally, PV loop area analysis can provide an evaluation of mechanical energies of a ventricular beat and an assessment of the LV efficiency.

To measure the ventricle volume, the conductance catheter technique has been used extensively, it is based on the electrical conductance of the blood contained in the cavity. This technique, nonetheless, is based on geometric assumptions, needs volume-dependent calibration and is limited by the non-linearity of the conductance-volume relation when large volume changes are involved Danton et al. [7]. Furthermore, it relies on Ohm's law which might not be fully appropriate due to the non-uniformity of the composition of ventricle and blood, and uses correction parameters that are error prone due to ventricle geometry and wall thickness changes during contraction Jacoby et al. [17]. Volume assessment using cine cardiac MR are proven to yield a more accurate and reliable estimate Jacoby et al. [17], Winter et al. [48]. For PV loop construction, simultaneous volume and pressure measurements are necessary, but given the magnetic nature of standard pressure sensors, this is problematic during MRI. The pressure signal would be contaminated by the MR environment and the presence of the sensor would produce artifacts on the image.

In this paper we present a feasibility study for simultaneous pressure measurements using optical sensors during MRI to produce valid indices for the assessment of LV function. In vivo measurements on one sheep were performed and pressure-volume loops were derived by combining MRI-estimated ventricular volumes with simultaneous on-site pressure measurements for various inotropic states. PV loops were analyzed and 
functional parameters were computed to evaluate the response to inotropic agents.

\section{Methods}

\subsection{Experimental Protocol \\ Animal preparation}

Two female sheep were used in this study. An intervention on the first sheep was performed to compare the optical pressure sensor measurements against the Millar standard probes. A subsequent intervention on another sheep allowed simultaneous pressure and volume measurements. The ewes were placed in a sitting position to induce reflex akinesia then anesthetized with a slow injection of $1 \mathrm{~g}$ of diluted thiopental. Tracheal tube was inserted and forced ventilation was started with a concentration of $1.5-2 \%$ isoflurane to maintain anesthesia. To prevent clotting, acetylsalicylic acid ( $0.5 \mathrm{~g})$ and heparin (3000 IU) were injected intravenously. The carotid artery was catheterized and a vascular dilator was inserted under $\mathrm{X}$-ray monitoring. The animals received humane care in compliance with the standards of the European Convention on Animal Care. The study was approved by a local institutional ethics committee (INRA, Paris, France). Qualified personnel supervised the procedures and adequate anesthesia was used to minimize unnecessary pain.

\section{In vivo pressure measurements}

An optical pressure sensor probe (model OPP-M, Opsens, Quebec, Canada) based on the white-light polarization interferometry technique was used. The probe tip, $0.4 \mathrm{~mm}$ in diameter and $0.5 \mathrm{~mm}$ in length, attached to a $10 \mathrm{~m}$ long optical fiber directly transduces pressure into an optical signal which is then sampled at $1 \mathrm{kHz}$. This device is fully MRI compatible, and immune to radiofrequency effects. The sensor is linear, with a total error of $2 \mathrm{mmHg}$ and a temperature sensitivity of $0.2 \mathrm{mmHg} /{ }^{\circ} \mathrm{C}$. Furthermore, each probe has two specific constant calibration factors. The nude fiber was sheathed into a non-magnetic $4 \mathrm{~F}$ catheter (C4F100D, Balt extrusion, Montmorency, France) $1 \mathrm{~m}$ in length. The sensor was positioned $5 \mathrm{~mm}$ before the catheter tip, facing a slot that had been manually cut in the catheter wall to ensure pressure transmission. The fiber was then glued into the catheter through another slot $5 \mathrm{~mm}$ above. A null point was set prior to inserting the sensor inside the sheep by zeroing the measurement at atmospheric pressure.

The validation of the optical probe against a reference pressure transducer (Millar, Houston, USA) was performed during an aortic intervention that served wider purposes than those stated in the present paper. During the intervention, the mounted optical probe and the Millar sensor were inserted simultaneously 
through the dilator up to the aorta of a $56 \mathrm{~kg}$ ewe. Pressure was recorded in vivo outside the magnet for about 5 minutes including 20 seconds during which ventilation was stopped to acquire a stable signal with no respiration modulation.

\section{Simultaneous in vivo pressure and volume measurements}

Pressure measurements with the optical probes were conducted simultaneously with MRI acquisitions in another female sheep $(60 \mathrm{~kg})$. After four acquisitions at a baseline condition (heart rate ranging between 78 and $83 \mathrm{bpm}$ ), the sheep was infused dobutamine at a rate of $5 \mu \mathrm{g} / \mathrm{kg} / \mathrm{min}$. Heart rate increased to $145-150$ $\mathrm{bpm}$ and three acquisitions were performed. Dobutamine rate was then decreased to $2.5 \mu \mathrm{g} / \mathrm{kg} / \mathrm{min}$ (heart rate decreased to $125-131 \mathrm{bpm}$ ) and a last acquisition was performed before waking the animal up. The whole MR procedure lasted 2 hours and 20 minutes.

\section{MRI acquisitions}

Cardiac imaging was performed with a 1.5 T MR scanner (Philips Medical Systems, Achieva) with a 5-element SENSE cardiac array coil and ECG triggering. After calibration and scouting sequences, true FISP gated cine images ("balanced TFE") were acquired in 12 sequential 8-mm short-axis slices (2 mm interslice gap) from the apex to the atrial-ventricular ring, for 30 cardiac phases ( $T E / T R=1.7 \mathrm{~ms} / 3.4 \mathrm{~ms}$, flip angle of $60^{\circ}$, field of view $=320 \times 240 \mathrm{~mm}^{2}$, matrix $=256 \times 192$, voxel size $1.25 \mathrm{~mm}$, echo train length of 10 , readout bandwidth $1042 \mathrm{~Hz}$ ). Mechanical ventilation was continued during the MRI acquisition procedures without respiratory synchronization. Other sequences were also acquired for other purposes, not mentioned here.

\subsection{Offline Pressure-Volume loop calculation}

For each slice, the LV was delineated semi-automatically and its area was computed then multiplied by the slice thickness to derive an elementary volume. These slice volumes were then added up to define the total LV volume. This was performed for all cardiac phases, and an LV volume evolution in time was obtained. During each of the imaging sessions the ventricular pressure was recorded continuously. Since we only have one volume curve reflecting a somewhat average volume evolution during the image acquisition, an average pressure cycle was derived for the loop computation. Figure 1 summarizes the cycle calculation. To delimit a heart cycle, pressure peaks were considered to be the end points of a pressure period. By 


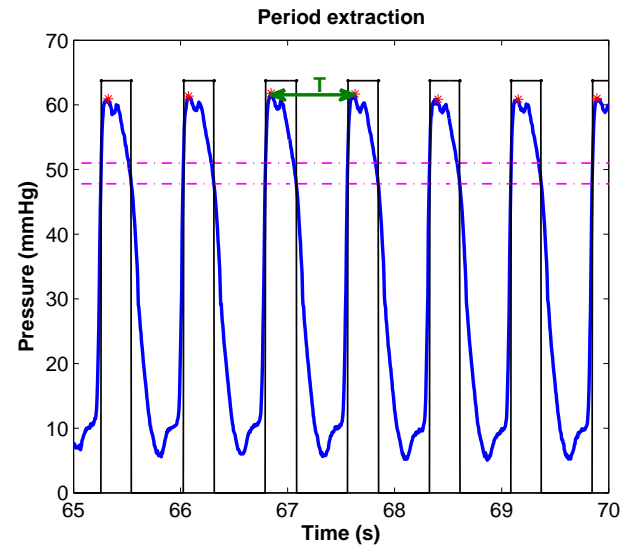

(a) Extraction of heart cycle

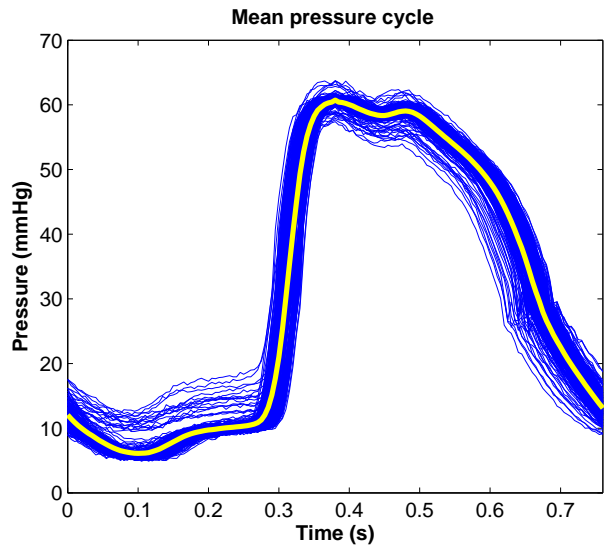

(b) Averaging over extracted periods

Figure 1: Mean pressure cycle computation

Local maxima were first identified at times $t_{p e a k, i}$, then individual periods $T_{i}$ were computed as being the times separating two consecutive peaks, finally pressure cycles were extracted between $t_{p e a k, i}-\frac{T_{i}}{2}$ and $t_{p e a k, i}+\frac{T_{i}}{2}$. A mean pressure curve was thus obtained by averaging all cycles.

applying a double threshold on the pressure signal, we delimited individual windows each containing a pressure peak. Afterward the local maximum was identified inside each window, thus defining a cycle end point. Once all peaks were determined, individual periods $T_{i}$ were computed, and cycles were extracted between $t_{p e a k, i}-\frac{T_{i}}{2}$ and $t_{p e a k, i}+\frac{T_{i}}{2}$. These cycles were then averaged to yield a mean pressure curve. The volume and pressure curves were then associated by assuming that maximum volume is attained with maximum $\frac{d P}{d t}$ Mason et al. [27].

\subsection{Pressure-Volume Analysis}

Selected indices were estimated using the PV loop in order to evaluate systolic and diastolic functions as well as mechanical efficiency during the three inotropic states. The end-systolic elastance was evaluated as a contractility index and the myocardium compliance was estimated during diastole to characterize the filling process. The effective arterial elastance, representing the afterload, was also evaluated to assess ventriculoarterial coupling. 
End-systolic elastance as contractility index

The end-systolic elastance $E_{e s}$ index is given by the slope of the end-systolic pressure-volume relationship (ESPVR). The ESPVR is defined as the line connecting the end-systolic points of a series of PV loops with different ventricular filling volumes. It is approximated as linear in the physiological range and is characterized by its slope, and a volume intercept $V_{0}$, a theoretical volume for which no pressure is developed Grossman et al. [14], Mehmel et al. [28], Suga et al. [41].

To obtain the ESPVR several PV loops are usually measured while gradually decreasing preload, using a balloon occlusion of the vena cava, for example. The invasive nature of this procedure, limiting clinical applications, has motivated several authors to propose methods for computing the ESPVR from a single PV loop. Takeuchi et al. Takeuchi et al. [44] simulated end-systolic isovolumic pressure by cosine fitting of the pressure curve, as done by Sunagawa et al. Sunagawa et al. [43], to locate a theoretical maximum pressure $P_{i s o}$ that would be attained if ejection did not take place. The $E_{e s}$ is then computed as the slope of the line connecting the point defined by $\left(E D V, P_{i s o}\right)$ to the end-systolic point on the PV loop. Other authors relied on a time-varying elastance model to derive $E_{e s}$ Senzaki et al. [36], Shishido et al. [37], Chen et al. [5]. Recently Brinke et al. Brinke et al. [3] using Takeuchi's technique with a modified fitting scheme (fifth order polynomial instead of a cosine), showed that a good estimation of intercept volumes can be achieved.

In this study we estimated the ESPVR following the method proposed by Brinke et al. Brinke et al. [3]. We computed a maximum isovolumic pressure $P_{\text {iso }}$ by using a fifth order polynomial fitting scheme to fit the left ventricle pressure curve. We started after end diastole and excluded from the fitting the pressure data points that lie after $d P / d t_{\max }$ and before $d P / d t_{\min }$ and those after the point where $d P / d t$ increased above $10 \%$ of $d P / d t_{\min }$

\section{LV compliance during the filling phase}

The pressure-volume relationship during diastole, known as the end-diastolic pressure-volume relationship (EDPVR), is generally exponential and depends on the elastic properties of the myocardium, the wall thickness and the ventricle's equilibrium volume $V_{0}$ Glantz and Kernoff [12] which is the intercept of the EDPVR with the volume axis. At any given instant of the EDPVR, the ventricle's stiffness modulus $E=\frac{\partial P}{\partial V}$ is given by the slope of the tangent to the curve at that point. We can also think in terms of compliance 
and compute $C=\frac{\partial V}{\partial P}$ as the slope reciprocal. The EDPVR is independent of the contractility state. When the inotropic state is enhanced (or decreased), the diastolic filling portion of the PV loop remains on this curve while sliding towards the left (or the right) Dickstein et al. [9], Thomas and Weyman [45]. This of course leads to compliance alterations in response to inotropic changes. In this study the EDPVR was fitted by $P=\alpha\left(e^{\beta\left(V-V_{0}\right)}-1\right)$, and the compliance was computed using the slope of the EDPVR during the slow filling phase, where viscous forces might be ignored, thus reflecting purely elastic properties of the ventricle. This phase is defined as the portion of diastole lying between the point where pressure starts increasing slowly while the volume continues to increase, and the point where pressure starts increasing rapidly while volume remains somewhat constant.

\section{Effective Arterial Elastance}

The arterial elastance is determined by the pressure in the ventricle at end systole, and by the amount of blood that the ventricle ejected into the arterial system such as $E_{a}=E S P / S V$, where ESP denotes the end-systolic pressure. It can be represented on the PV loop diagram as the line connecting the end systolic coordinates and the end-diastolic volume point on the volume axis.

\section{Cardiac work efficiency}

The efficiency of energy transfer from the ventricle to the arterial system, called cardiac work efficiency (CWE), is given as the ratio of the stroke work to the total mechanical energy, $C W E=S W / P V A$. The total mechanical energy of a ventricular beat known as the pressure volume area (PVA) is equal to the sum of potential energy and stroke work, $P V A=P E+S W$,Suga [40]. The stroke work (SW) is given by the area inside the PV loop, and the potential energy (PE) is represented by the area of the triangle between the point of end systole on the PV loop, $V_{0}$ and the end-systolic volume (ESV) intercept on the EDPVR.

CWE is reciprocally related to $\frac{E_{a}}{E_{e s}}$ Kameyama et al. [18], it increases with contractility enhancement, and decreases with afterload increase Nozawa et al. [30].

\section{Results}

\subsection{In vivo pressure measurements validation}

Figure 2 shows two 20 second extracts of the recorded pressure signals in the aorta outside the MRI, one while the ventilation was off and another, almost 2 minutes later during normal ventilation. The Opsens 

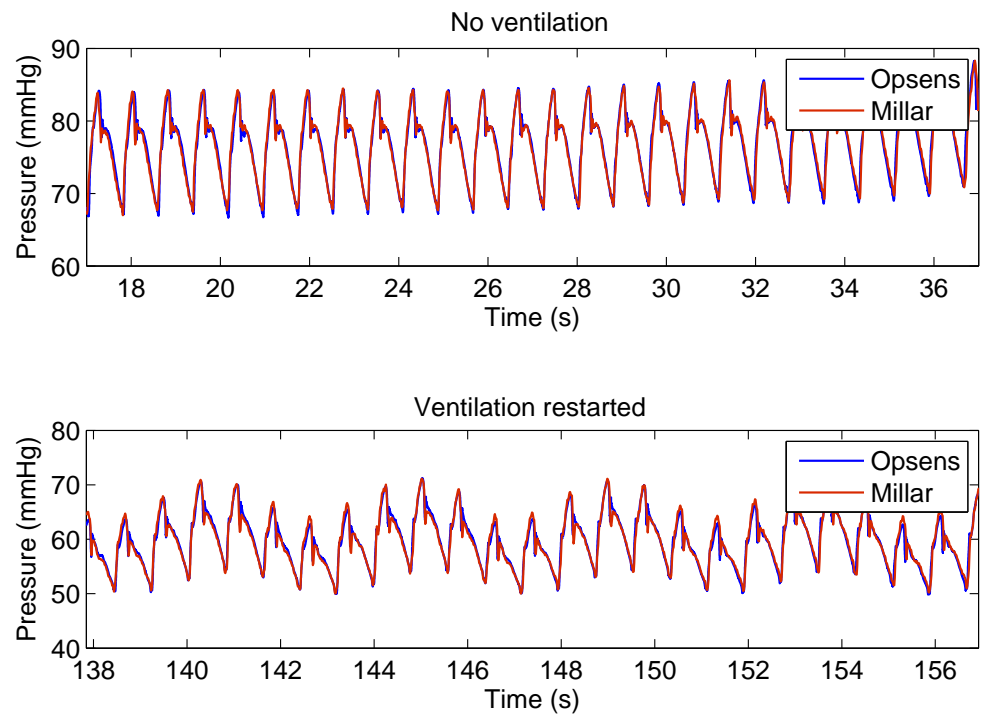

Figure 2: Opsens and Millar pressure signals

Extracts of pressure signals recorded simultaneously using OpSens and Millar are represented during a time period where the ventilation had been stopped, and later when the ventilation had been restarted. The OpSens offset was corrected so that the mean error would be null.

offset was corrected such that the mean error would be zero. Figure 3 shows the extracted average cycles of both Opsens and Millar with no ventilation. Pressure measured with the optical transducer agrees well with the standard Millar sensor.

\subsection{MRI acquisitions and PV loops calculations}

As shown in figure 4 the images were not affected by the presence of the pressure sensors, however some artifacts due to breathing motion can be noted. Pressure-volume loops were computed for all measurements covering three contractility states, figure 5 shows an example of the computed pressure and volume curves for a baseline state. Overall we obtained 8 PV loops : 4 for baseline, 1 with dobutamine 2.5, and 3 with dobutamine 5 .

\subsection{Baseline measurements}

The computed mean baseline parameters were compared to values obtained in other studies dealing with sheep Charan et al. [4], Lee et al. [23], Bauer et al. [2], Ratcliffe et al. [33], Pilla et al. [32], Segers 


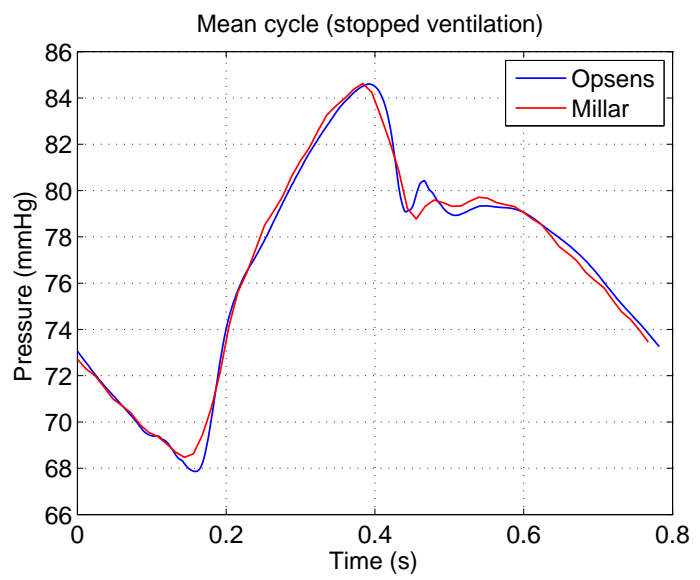

Figure 3: Opsens and Millar average pressure cycles The mean pressure cycles of Opsens and Millar extracted during the period when the ventilation was stopped.

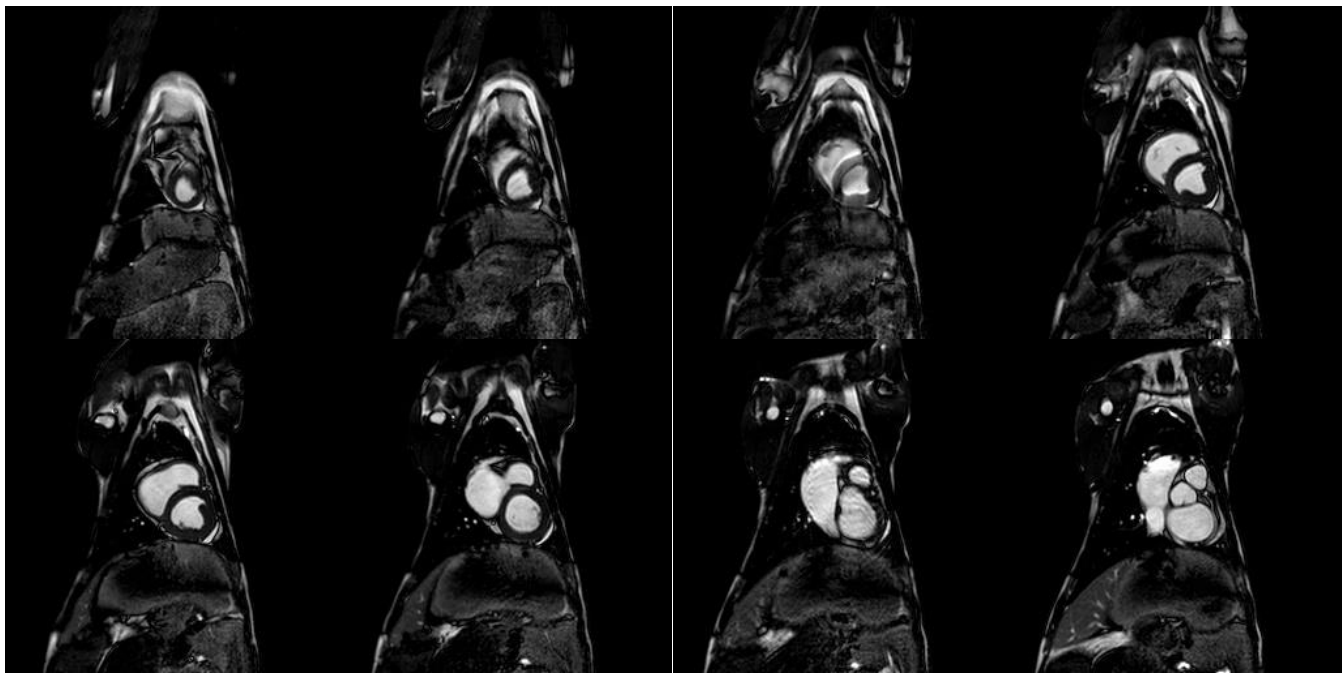

Figure 4: Left ventricle short axis slices acquired with MRI at end systole MRI images used for LV volume estimation were not affected by the optical sensors but show some breathing motion artifacts. 
Pressure and Volume curves

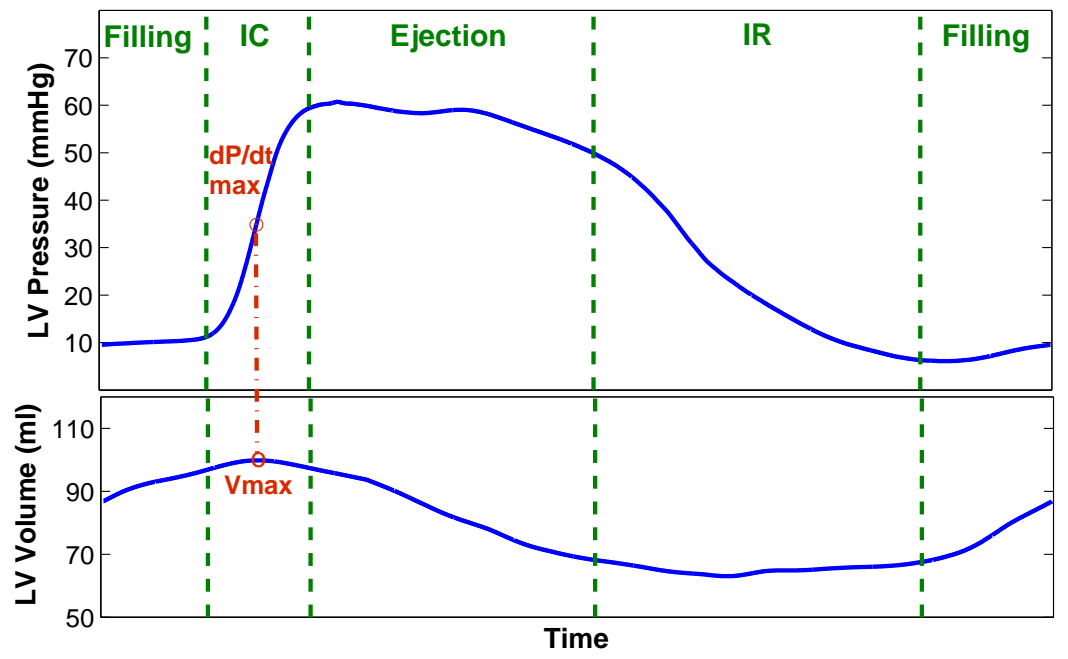

Figure 5: Associated LV pressure and volume curves

Measured volume and average pressure cycle during baseline state. To produce the PV loop, the curves were matched so that maximum volume would coincide with the maximum first derivative of pressure.

et al. [35] in order to situate our calculations with respect to usual parameter values. These values are given in table 1 , shown as (mean value \pm standard deviation)

\subsection{Contractility states comparison}

Figure 6 shows the experimental time-line and heart rate evolution throughout the consecutive states. The instantaneous frequencies were computed, over the pressure recordings, as the inverse of the time interval separating two pressure peaks, as shown in figure 1a. Heart rate (HR) increases gradually after the dobutamine administration starts, it stabilizes then decreases when the dobutamine rate is diminished. An HR increase can be observed as we go from one rate to another, as the remaining dobutamine might have been injected rapidly when flushing the tubes for the next infusion. Of course, functional changes in response to the inotropic agents, go beyond the obvious HR variation. Not only did the pressure cycle period vary, but its shape was drastically altered. Figure 7 shows mean pressure cycles, each representing an inotropic state.

Pressure volume loop analysis was performed for all 8 measurements. The obtained parameter values are given in table 2. Compared to baseline, $E_{e s}$ increased significantly with positive inotropic stimuli. Figure 


\begin{tabular}{|c|c|c|c|c|c|c|c|}
\hline & Present Study & Charan et al. [4] & Lee et al. [23] & Bauer et al. [2] & Ratcliffe et al. [33] & Pilla et al. [32] & Segers et al. [35] \\
\hline Weight (kg) & 70 & 72.50 & $35 \pm 5$ & & $41.62 \pm 0.45$ & & $40, .00 \pm 5.00$ \\
\hline$H R(\mathrm{bpm})$ & $79.28 \pm 2.79$ & $97 \pm 5$ & $85 \pm 21$ & $109 \pm 15$ & $102.10 \pm 14.50$ & $97.60 \pm 9.73$ & 81 \\
\hline$C W E(\%)$ & $76.26 \pm 4.23$ & & & & & $60.80 \pm 12$ & \\
\hline$E D V(\mathrm{ml})$ & $95.22 \pm 3.82$ & & & & & 60 & 79.00 \\
\hline$E S P(\mathrm{mmHg})$ & $48.04 \pm 3.58$ & & & $87 \pm 34$ & $100.30 \pm 12.80$ & & \\
\hline $\operatorname{ESV}(\mathrm{ml})$ & $68.55 \pm 2.49$ & & & & & $36.15 \pm 9.78$ & \\
\hline$P_{\max }(\mathrm{mmHg})$ & $59.46 \pm 1.53$ & & & & $102.30 \pm 12$ & & \\
\hline$V_{0}(\mathrm{ml})$ & $38.49 \pm 3.36$ & & & & -27.69 & $-24.80 \pm 20.10$ & -36.00 \\
\hline$S V(\mathrm{ml})$ & $26.67 \pm 1.84$ & & & & $24.60 \pm 4.80$ & $20.20 \pm 8.76$ & $49.00 \pm 6.00$ \\
\hline$E F(\%)$ & $27.99 \pm 1.16$ & & & $60 \pm 9$ & $57.60 \pm 3.50$ & 35 & \\
\hline$E D P(\mathrm{mmHg})$ & $10.88 \pm 0.87$ & $7 \pm 1.60$ & 7.30 & $9 \pm 3$ & $13.80 \pm 5.20$ & $5.25 \pm 1.86$ & \\
\hline$C(\mathrm{ml} / \mathrm{mmHg})$ & $6.23 \pm 0.47$ & & 2.94 & & $1.70 \pm 0.65$ & & \\
\hline$C O(\mathrm{I} / \mathrm{min})$ & $2.11 \pm 0.11$ & $4 \pm 0.40$ & & $2 \pm 0.30$ & $2.45 \pm 0.24$ & $2.03 \pm 0.71$ & \\
\hline$E_{e s}(\mathrm{mmHg} / \mathrm{ml})$ & $1.62 \pm 0.19$ & & 0.46 & $2.71 \pm 0.35$ & $2.52 \pm 0.27$ & 3.40 & 1.37 \\
\hline$S W(\mathrm{mmHg} \cdot \mathrm{ml})$ & $1574.27 \pm 183.24$ & & & & & 1400 & \\
\hline$\frac{d p}{d t_{\max }}(\mathrm{mmHg} / \mathrm{s})$ & $1020.64 \pm 20.24$ & $1902 \pm 267$ & & $1346 \pm 478$ & & $1492 \pm 235$ & \\
\hline$\frac{d p}{d t} \min (\mathrm{mmHg} / \mathrm{s})$ & $-347.02 \pm 20.26$ & & & & & $-1813 \pm 359$ & \\
\hline$P V A(\mathrm{mmHg} \cdot \mathrm{ml})$ & $2066.15 \pm 238.61$ & & 2558 & & & 3400 & \\
\hline$P E(\mathrm{mmHg} \cdot \mathrm{ml})$ & $491.89 \pm 107.13$ & & & & & $1024 \pm 345$ & \\
\hline
\end{tabular}

Table 1: Comparison of PV loop extracted parameters with respect to the literature

8 shows an example of PV loops representing the three states (baseline measurement $n^{\circ} 4$, dobutamine 2.5, dobutamine 5 measurement $n^{\circ} 2$ ), as well their corresponding end-systolic elastances. Moreover, the cardiac work efficiency was enhanced by dobutamine administration. As expected, the diastolic compliance increased with inotropic enhancement. As contractility is increased, the PV loops shift to the left on the EDPVR, thus going towards lower slopes (i.e. stiffness).

\section{Discussion}

MRI is considered to be the gold standard for absolute cardiac volume measurements. Obtaining PV loops requires simultaneously recording pressure during the MRI sessions. Pattynama et al.Pattynama et al. [31] used a Millar micromanometer to achieve such measurements, and argued that because it is made from brass the obtained pressure signals were not significantly altered, however, some image artifacts were observed. Schmitt et al.Schmitt et al. [34] and Kuehne et al.Kuehne et al. [22] used liquid filled catheters to measure ventricular pressure down a $1 \mathrm{~m}$ pressure line. The impedance of the transmission line reduces pressure amplitudes, and constitutes a low pass filter that attenuates high frequency components. A pressure sensor placed inside the ventricle would provide a more accurate representation of the actual LV pressure. The use of optical sensors in this study enabled pressure measurements directly in the ventricle without any interaction with the MRI acquisition. 

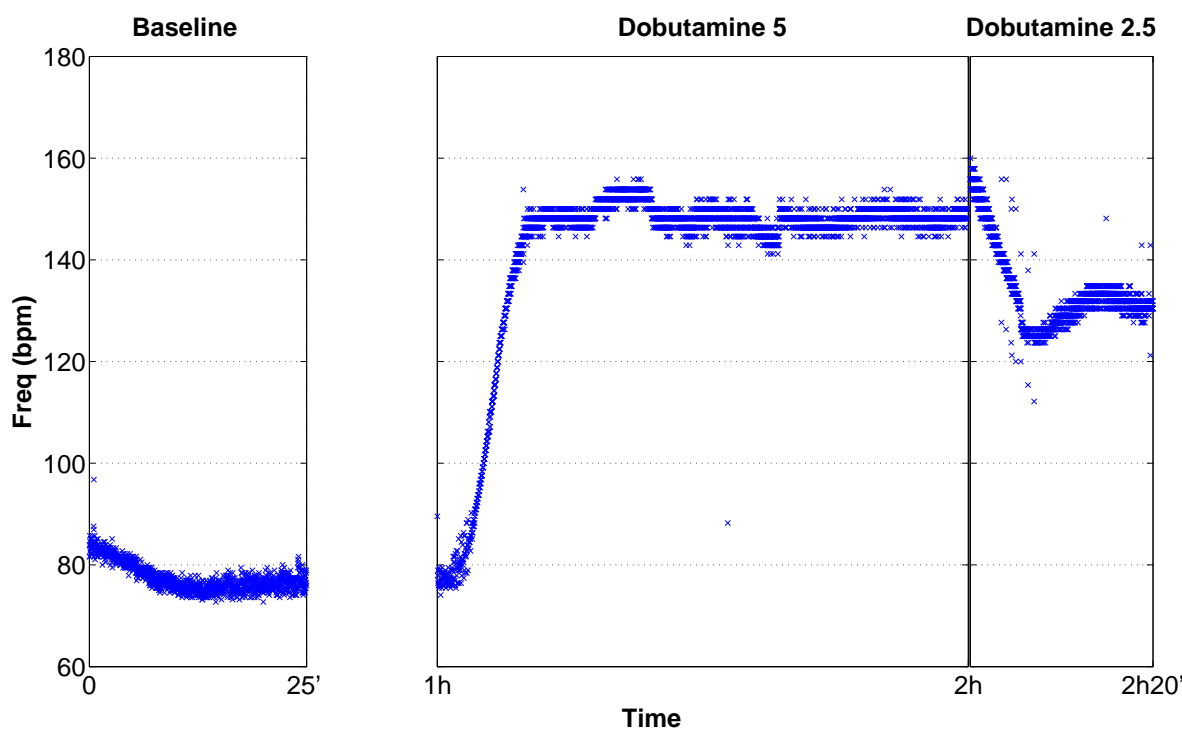

Figure 6: Heart rate evolution throughout the experiment

Heart rate evolution vs the experimental time-line throughout the three consecutive inotropic states.

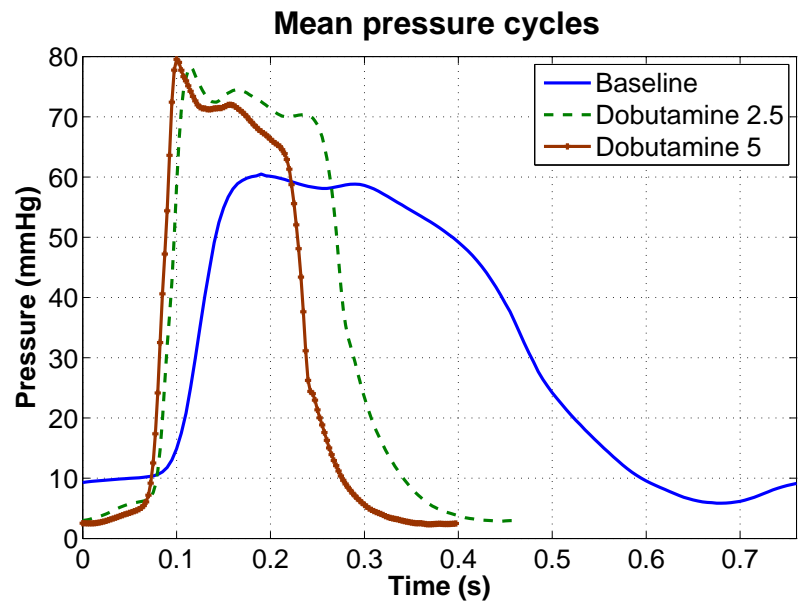

Figure 7: Mean pressure cycles for the three inotropic states

Extracted average cycles for baseline, dobutamine $2.5 \mu \mathrm{g} / \mathrm{kg} / \mathrm{min}$ and dobutamine $5 \mu \mathrm{g} / \mathrm{kg} / \mathrm{min}$. The shape and duration of the pressure cycle is altered in response to positive inotropic stimuli. 


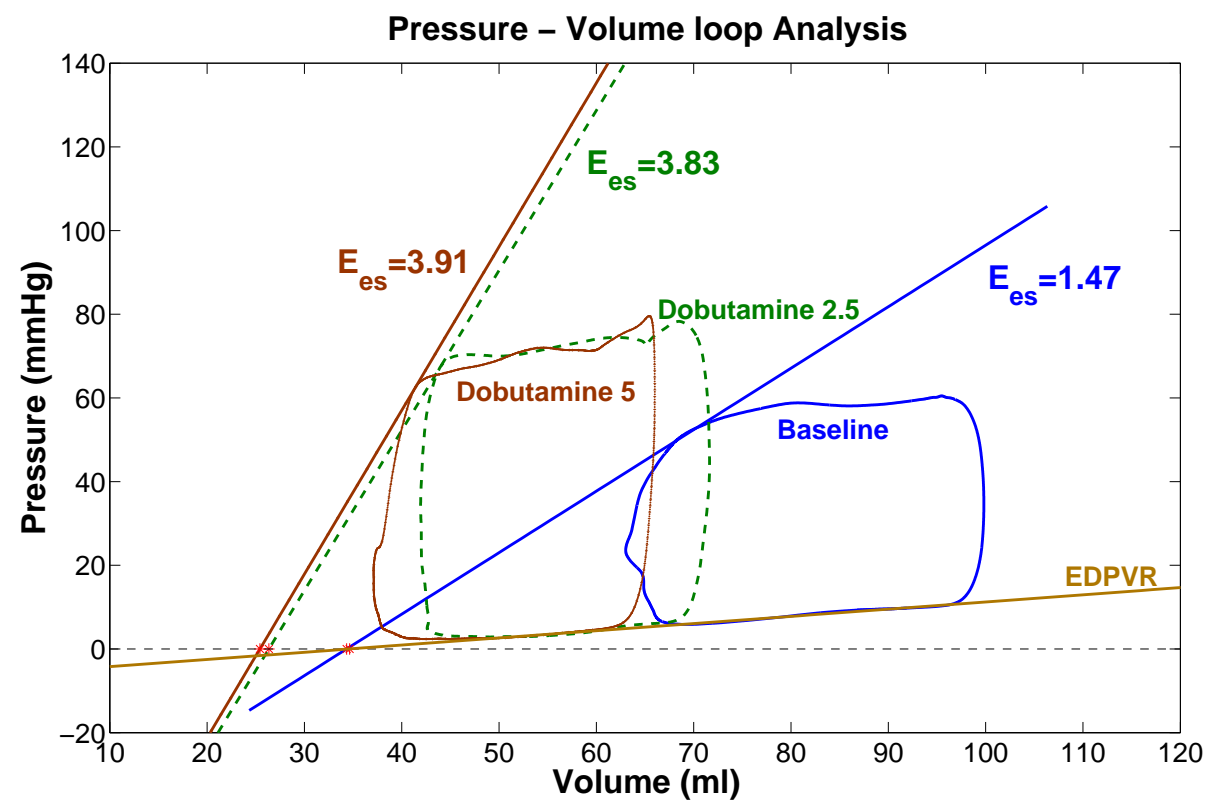

Figure 8: End-systolic elastance for the three contractility states

PV loops obtained during baseline, dobutamine $2.5 \mu \mathrm{g} / \mathrm{kg} / \mathrm{min}$ and dobutamine $5 \mu \mathrm{g} / \mathrm{kg} / \mathrm{min}$. For each loop ESPVR is represented and the value of its slope $E_{e s}$ is given in $\mathrm{mmHg} / \mathrm{ml}$.

\begin{tabular}{|c|c|c|c|c|c|c|c|c|}
\hline \multirow[b]{2}{*}{$H R$} & \multirow[b]{2}{*}{$(b p m)$} & \multicolumn{3}{|c|}{ Baseline } & \multirow{2}{*}{$\begin{array}{r}\text { Dobutamine } 2.5 \\
129.77 \\
\end{array}$} & \multicolumn{3}{|c|}{ Dobutamine 5} \\
\hline & & 78.28 & \pm & 2.79 & & 150.99 & \pm & 0 \\
\hline$S V$ & $(m L)$ & 26.67 & \pm & 1.84 & 25.39 & 20.59 & \pm & 1.40 \\
\hline$E F$ & $(\%)$ & 27.99 & \pm & 1.16 & 36.66 & 33.14 & \pm & 2.88 \\
\hline$P_{\max }$ & $(\mathrm{mmHg})$ & 59.46 & \pm & 1.53 & 78.31 & 77.64 & \pm & 2.52 \\
\hline$C$ & $(\mathrm{~mL} / \mathrm{mmHg})$ & 6.23 & \pm & 0.47 & 6.88 & 8.55 & \pm & 3.35 \\
\hline$E_{e s}$ & $(m m H g / m L)$ & 1.62 & \pm & 0.19 & 3.83 & 4.21 & \pm & 0.26 \\
\hline$\overline{V_{0}}$ & $(m L)$ & 38.49 & \pm & 3.36 & 26.35 & 28.26 & \pm & 6.59 \\
\hline$\frac{d P}{d t} \max$ & $(\mathrm{mmHg} / \mathrm{s})$ & 1020.64 & \pm & 20.24 & 3370.92 & 3556.28 & \pm & 232.13 \\
\hline$\frac{d P}{d t} \min$ & $(\mathrm{mmHg} / \mathrm{s})$ & -347.02 & \pm & 20.26 & -1860.39 & -2342.00 & \pm & 428.75 \\
\hline$S W$ & $(m m H g . m L)$ & 1574.27 & \pm & 183.24 & 1939.42 & 1548.46 & \pm & 229.35 \\
\hline$C W E$ & $(\%)$ & 76.26 & \pm & 4.23 & 80.91 & 88.38 & \pm & 3.24 \\
\hline$E_{a}$ & $(m m H g / m L)$ & 1.81 & \pm & 0.18 & 2.64 & 2.74 & \pm & 0.37 \\
\hline$E_{a} / E_{e s}$ & & 1.12 & \pm & 0.11 & 0.69 & 0.65 & \pm & 0.10 \\
\hline
\end{tabular}

Table 2: Functional indices extracted from the PV loops of the three inotropic states 


\subsection{Volume measurements}

Unlike the conductance catheter technique, PV loop calculation using MRI imposes the use of an average pressure cycle vs average volume instead of instantaneous pressure vs instantaneous volume. Thus, the PV loop here reflects a global performance cycle and does not take into account an exact punctual correspondence between individual volume and a pressure measurements. Moreover the time synchronization between the volume and pressure cycle in this study was done based on bibliographic data, a more accurate match could be achieved by using the pressure signal to trigger the image acquisition and thus synchronize both waveforms.

Due to the lack of real-time volume recordings, it was impossible to obtain a series of load varying PV loops using MRI because a steady state over several cycles is needed in order to compute a volume. For the same reason, the assessment of contractility transit states is not possible using MRI. This remains, for now, an advantage in favor of the conductance technique, however, future advances in MRI may enable the overcoming of this limitation.

\subsection{Pressure measurements}

Even though the dynamics of the pressure measurements performed here were shown to be accurate, these optical pressure sensors present an offset that drifts throughout the experiment, thus introducing an unknown pressure shift between measurements. This variable drift can exceed $2 \mathrm{mmHg} /$ hour. To be able to compare the obtained loops and the extracted parameters, a correction was necessary to bring the pressure cycles to a common reference. To eliminate these relative offsets we relied on theoretical fact that the filling parts of all measured PV loops should lie on the same EDPVR. Hence, we computed that relation for one of the baseline loops (we arbitrarily chose the fourth) and then shifted the pressure cycles of the rest of the loops so that they would be supported by the estimated EDPVR. Of course this does not eliminate the "absolute" pressure offset that is added to the reference pressure measurement, but at least all loops had that same offset and could be compared. An attempt to estimate and correct the absolute offset was made by considering the EDPVR and ESPVR intersection point. Theoretically these relationships should have the same zero axis intercept. Thus, we could assume that the absolute offset would be eliminated by shifting the reference baseline loop so that EDPVR and ESPVR intersect at zero pressure. Of course the same shift would afterward be applied to the rest of the loops. This procedure would, however, not 
guarantee the elimination of the real offset. In fact it greatly depends on the ESPVR estimation, which itself is not very precise given that it is computed from a single beat. Besides given the instability of ESPVR and the equilibrium volume $V_{0}$ and their variability between repeated measurements Spratt et al. [38], the chosen loop probably does not reflect the real $V_{0}$.

To sum up, the presented pressures here might not actually reflect the real ones, but present a residual offset that introduces errors in the calculations of some parameters, such as $V_{0}, P E$, and $E_{a}$. Other parameters such as $E_{e s}, C$, and $S W$, are independent of the pressure offset and thus can be assumed accurate even if there's an unknown shift of the PV loop. Regardless of whether the offset is substantial or not, comparison between the considered contractility states remains valid, since the relative offsets are corrected and the absolute shift applies equally to all states. Further studies will focus on modeling the sensors behavior to enable measurement correction.

\subsection{Baseline PV loops indices}

When comparing baseline measurements with other authors works we found that some parameters such as $E_{e s}$ agree well with the literature range. Others, like the compliance and $V_{0}$, differ significantly from what is obtained by other authors. These differences can be explained by the fact that the sheep studied by those authors are much smaller (weighting around $40 \mathrm{~kg}$ ) than the one used in our experiments $(60 \mathrm{~kg})$. Since the diastolic compliance decreases with the chamber size Diamond et al. [8], Forrester et al. [10], smaller animals with smaller hearts have less compliant ventricles. Moreover, smaller volumes mean that the PV loops are shifted to the left, thus the ESPVR axis intercept is also shifted to the left, thus explaining the smaller $V_{0}$.

\subsection{Response to inotropic stimuli}

Both end systolic elastance and diastolic compliance increased significantly in response to positive inotropic stimuli. Increasing the dobutamine concentration did not enhance the stroke work (the SV decreased with an insignificant increase in maximum pressure), it did however somewhat enhance the cardiac work efficiency. The SW varies very slightly with afterload but is very sensitive to preload Kass et al. [19] , and thus the study of the relationship between the EDV and the SW would be more suitable. This relationship is called the preload recruitable stroke work (PRSW). It is a linear relationship Glower 
et al. [13] which slope reflects the inotropic state. It was also shown to be insensitive to afterload over the physiological range, and thus qualifies as a contractility index, although it might depend on cardiac geometry and heart rate Glower et al. [13]. Nonetheless, the PRSW is less sensitive to inotropic changes than $E_{e s}$ and $d P / d t_{\max } \mathrm{vs} E D V$ Little et al. [25]. Since preload conditions variations were not performed during this feasibility study, it was not possible to derive such relationships which rely on multiple PV loops. A method to estimate the slope of PRSW from a single beat was proposed by Mohanraj et al. Mohanraj and Feneley [29], however their method did not exclude prior preload variation experiments. For their estimations they computed an empirical constant from a set of VCO experiments, which they used for the slope estimation of another group of the same species.

The ESPVR axis intercept $V_{0}$ varies slightly among states but not distinctly enough to be attributed to contractility changes. This volume should be independent of the inotropic state Suga et al. [41], all ESPVR and EDPVR should meet at the same axis intercept. Positive inotropic interventions would increase $E_{e s}$ and shift the ESPVR to the left, while depressors of the cardiac function would decrease $E_{\text {es }}$ shifting ESPVR to the right, nevertheless the volume axis intercept $V_{0}$ would not significantly change Spratt et al [38], Dickstein et al. [9]. The changes observed here account for the instability of $V_{0}$ Spratt et al. [38] as well as measurement and estimation errors. Note that the $V_{0}$ range here is affected by the pressure offset and its correction.

The effective arterial elastance $E_{a}$ increases with inotropic enhancement as end systolic pressure (ESP) is increased. The ventriculoarterial coupling index $E_{a} / E_{e s}$ decreases when contractility increases. Studies have shown that $\frac{E_{a}}{E_{e s}}=0.5$ ensures maximal CWE Starling [39], Asanoi et al. [1]. On the other hand, given that the energy transfer from one elastic chamber to another is maximal when they both have the same elastance Sunagawa et al. [42], maximal SW would occur when $E_{a}=E_{e s}$. It has been shown that the normal heart at rest operates at neither maximal CWE nor maximal SW, but in fact finds an optimal working point between the two, which is actually closer to maximal efficiency Starling [39], Asanoi et al. [1]. In our baseline measurements, $E_{a} / E_{e s}$ is close to 1 , indicating that the ventricle is working near maximal SW. With dobutamine, this index is approximately 0.6 , thus the heart is functioning around the optimal working point between maximal SW and maximal CWE, while remaining closer to maximal efficiency. 


\subsection{Valid contractility index}

The results obtained here showed that parameters computed using volume alone might not correctly reflect LV contractility. In fact, the stroke volume measurements confirmed that it is not a good contractility index. Despite the contractility enhancement, because of its load dependence, the stroke volume of the dobutamine loop is smaller than that of baseline. Since the preload (EDV) decreased, so has the SV. The same can be argued for the ejection fraction which did not reflect the inotropic increase induced by the dobutamine augmentation.

On the other hand, $d P / d t_{\max }$ often used as a contractility index based on pressure measurements alone, behaved as expected in this study and did increase with inotropic stimuli. Even though this index is more sensitive to inotropic changes than $E_{e s}$, it depends on heart rate, and is still preload-dependent. Even when considering $d P / d t_{\text {max }}$ vs $E D V$ to negate the preload effect, it was shown that $E_{e s}$ remains a better index. Kass et al. found that in the presence of both preload and afterload alterations, $E_{e s}$ was relatively less affected than $d P / d t_{\max } \mathrm{vs} E D V$. So, despite the $E_{e s}$ lesser sensitivity to inotropic change, its minimal dependence on both types of load alterations, coupled with its adequate characterization of contractility, make it somewhat more advantageous than $d P / d t_{\max }$ vs $E D V$ Kass et al. [19].

The accuracy and load independence of $E_{e s}$ is however questionable when obtained using single-beat estimation methods which rely on load-dependent elements such as $d P / d t$ and end-diastolic volume. Sincepreload has not been changed during this study and only single beat methods were used, it would be more valid to view the estimated $E_{e s}$ as a load-dependent approximation of the load-independent elastance Kjorstad et al. [21]. Even though these methods might underestimate $E_{e s}$ and do not agree closely with the vena cava occlusion (VCO) technique estimation, they are still reasonable approaches given their experimental advantages Brinke et al. [3].

\section{Conclusion}

By analyzing the ventricular PV loop, several functional parameters can be extracted and used to assess the LV performance. In this study we showed the feasibility of PV loop computation using simultaneous MRI volume estimation and direct pressure measurements with optical sensors on a sheep in three inotropic states. The optical nature of the pressure sensors ensured the absence of pressure signal contamination as 
well as image artifacts. Functional parameters were extracted and compared to the values reported in the literature showing good agreement. Contractility indices such as end diastolic elastance, were possible to estimate despite the pressure sensors offset problem. Following up on this feasibility investigation, a more comprehensive study including several animals will be carried out.

\section{List of abbreviations}

CO Cardiac Output

CWE Cardiac Work Efficiency

EDPVR End-Diastolic Pressure-Volume Relationship

EDV End Diastolic Volume

EF Ejection Fraction

ESP End Systolic Pressure

ESPVR End-Systolic Pressure-Volume Relationship

ESV End-Systolic Volume

HR Heart Rate

IC Isovolumic Contraction

IR Isovolumic Relaxation

LV Left Ventricle

MRI Magnetic Resonance Imaging

PE Potential Energy

PV Pressure-Volume

PVA Pressure Volume Area 
SV Stroke Volume

SW Stroke Work

VCO Vena Cava Occlusion

\section{Competing interests}

The authors declare that they have no competing interest.

\section{Declarations on funding and ethical approval}

There was no funding for this research. This study did not involve any human subjects.

\section{Authors contributions}

DAR: participated in data acquisition, performed data processing, analysis and interpretation, and drafted the manuscript. ED: participated in the conception of the study, performed critical revision of the manuscript, and participated in data acquisition. LdR: participated in data acquisition. YB: participated in animal preparation and data acquisition. EM: participated in the conception of the study, and animal preparation. All authors read and approved the final manuscript

\section{Acknowledgment}

The authors would like to thank Alain De Cesares with the Laboratoire d'Imagerie Fonctionnelle, U678, Paris, France, for his contribution to a part of data processing.

\section{References}

[1] Asanoi, H., Sasayama, S., Kameyama, T., 1989. Ventriculoarterial coupling in normal and failing heart in humans. Circ. Res. 65, 483-493.

[2] Bauer, F., Jones, M., Shiota, T., Firstenberg, M., Qin, J., Tsujino, H., Kim, Y., Sitges, M., Cardon, L., Zetts, A., Thomas, J., OCT 2 2002. Left ventricular outflow tract mean systolic acceleration as a surrogate for the slope of the left ventricular end-systolic pressure-volume relationship. J. Am. Coll. Cardiol. 40 (7), 1320-1327. 
[3] Brinke, E., Klauts, R., Verwey, H., van der Wall, E., Dion, R., Steendijk, P., 2010. Single-beat estimation of the left ventricular end-systolic pressure-volume relationship in patients with heart failure. Acta Physiol. 198, 37-46.

[4] Charan, N., Ripley, R., Carvalho, P., MAY 1998. Effect of increased coronary venous pressure on left ventricular function in sheep. Resp. Physiol. 112 (2), 227-235.

[5] Chen, C., Fetics, B., Nevo, E., Rochitte, C., Chiou, K., Ding, P., Kawagachi, M., Kass, D., 2001. Nonunvaasive single-beat determination of left ventricular end-systolic elastance in humans. J. Am. Coll. Cardiol. 38, 2028-2034.

[6] Cohn, P., Liedtke, A., Serur, J., Sonnenblick, E., 1972. Maximal rate of pressure fall (peak negative $\mathrm{dp} / \mathrm{dt}$ ) during ventricular relaxation. Cardiovasc. Res. 6, 263-267.

[7] Danton, M., Greil, G., Byrne, J., Hsin, M., Cohn, L., Maier, S., 2003. Right ventricular volume measurement by conductance catheter. Am. J. Physiol.-Heart C. 285, 1774-1785.

[8] Diamond, G., Forrester, J., Hargis, J., Parmley, W., Danzig, R., J., S., 1971. Diastolic pressure-volume relationship in the canine left ventricle. Circ. Res. 29, 267-275.

[9] Dickstein, M., Yano, O., Spotnitz, H., Burkhoff, D., 1995. Assessment of right ventricular contractile state with the conductance catheter technique in the pig. Cardiovasc. Res. 29, 820-826.

[10] Forrester, J., Diamond, G., Parmley, W., J., S., 1972. Early increase in left ventricular complinace after myocardial infarction. J. Clin. Invest. 51, 598-603.

[11] Gaddam, K., Oparil, S., 2009. Diastolic dysfunction and heart failure with preserved ejection fraction: rationale for raas antagonist/ccb combination therapy. Am J Hypertens 3 (1), $52-68$.

[12] Glantz, S., Kernoff, R., 1975. Muscle stiffness determined from canine left ventricular pressure- volume curves. Circ. Res. 37, 787-794.

[13] Glower, D., Spratt, J., Snow, N., Kabas, J., Davis, J., Olsen, C., Tyson, G., D., S., Rankin, J., 1985. Linearity of the frank-starling relationship in the intact heart : the concept of preload recruitable stroke work. Circulation 71, 994-1009. 
[14] Grossman, W., Braunwald, E., Mann, T., Mclaurin, L., Green, L., 1977. Contractile state of the left ventricle in man as evaluated from end-systolic pressure-volume relations. Circulation 56, 845-852.

[15] Grossman, W., Haynes, F., Paraskos, J., Saltz, S., Dalen, J., Dexter, L., 1972. Alterations in preload and myocardial mechanics in the dog and in man. Circ. Res. 31, 83-94.

[16] Hayashida, K., Sunagawa, K., Noma, M., Sugimachi, M., Ando, H., Nakamura, M., 1992. Mechanical matching of the left ventricle with the arterial system in exercising dogs. Circ. Res. 71, 481-489.

[17] Jacoby, C., Molojavyi, A., Flogel, U., Merx, M., Ding, Z., Schrader, J., JAN 2006. Direct comparison of magnetic resonance imaging and conductance microcatheter in the evaluation of left ventricular function in mice. Basic Res. Cardiol. 101 (1), 87-95.

[18] Kameyama, T., Asanoi, H., Ishizaka, S., Yamanishi, K., Fujita, M., Sasayama, S., 1992. Energy conversion efficiency in human left ventricle. Circulation 85, 988-996.

[19] Kass, D., Maughan, W., Guo, Z., Kono, A., Sunagawa, K., Sagawa, K., 1987. Comparative influence of load versus inotropic states on indexes of ventricular contractility: experimental and theoretical analysis based on pressure-volume. Circulation 76, 1422-1436.

[20] Kelly, R., Ting, C., Yang, T., Liu, C., Maughan, W., Chang, M., Kass, D., 1992. Effective arterial elastance as index of arterial vascular load in humans. Circulation 86, 513-521.

[21] Kjorstad, K., Korvald, C., Myrmel, T., 2001. Pressure-volume-based single-beat estimations cannot predict left ventricular contractility in vivo. Am. J. Physiol.-Heart C. 282, 1739-1750.

[22] Kuehne, T., Yilmaz, S., Steendijk, P., Moore, P., Groenink, M., Saaed, M., Weber, O., Higgins, C., Ewret, P., Fleck, E., Nagel, E. Schulze-Neick, I., Lange, P., 2004. Magnetic resonance imaging analysis of right ventricular pressure-volume loops: In vivo validation and clinical application in patients with pulmonary hypertension. Circulation 110, 2010-2016.

[23] Lee, L. S., Ghanta, R. K., Mokashi, S. A., Coelho-Filho, O., Kwong, R. Y., Bolman, III, R. M., Chen, F. Y., APR 2010. Ventricular restraint therapy for heart failure: The right ventricle is different 
from the left ventricle. J. Thorac. Cardiovasc. Surg. 139 (4), 1012-1018, 35th Annual Meeting of the Western-Thoracic-Surgical-Association, Banff, CANADA, JUN 24-27, 2009.

[24] Little, W., 1985. The left ventricular dp/dt max -end-diastolic volume relation in closed-chest dogs. Circ. Res. 56, 808-815.

[25] Little, W., Cheng, C., Mumma, M., Igarashi, Y., Vinten-Johansen, J., Johnston, W., 1989. Comparison of measures of left ventricular contractile performance derived from presure-volume loops in conscious dogs. Circulation 80, 1378-1387.

[26] Maeder, M., Kaye, D., 2009. Heart failure with normal left ventricular ejection fraction. J. Am. Coll. Cardiol. 53 (11), $905-918$.

[27] Mason, D., Braunwald, E., Covell, J., Sonnenblick, E., Ross, J., 1971. Assessment of cardiac contractility: The relation between the rate of pressure rise and ventricular pressure during isovolumic systole. Circulation 44, 47-58.

[28] Mehmel, H., Stockins, B., Ruffmann, K., von Olshausen, K., Schuler, G., Kubler, W., 1981. The linearity of the end-systolic pressure-volume relationship in man and its sensitivity for assessment of left ventricular function. Circulation 63, 1216-1222.

[29] Mohanraj, K., Feneley, M., 2000. Single-beat determination of preload recruitable stroke work relationship:derivation and evaluation in conscious dogs. J Am Coll Cardiol. 35, 502-513.

[30] Nozawa, T., Yasumura, Y., Futaki, S., Tanaka, N., Uenishi, M., Suga, H., 1988. Efficiency of energy transfer from pressure-volume area to external mechanical work increases with contractile state and decreases with afterload in the left ventricle of the anesthetized closed-chest dog. Circulation 77 , $1116-1124$.

[31] Pattynama, P., Deroos, A., Vandervelde, E., Lamb, H., Steendijk, P., Hermans, J., Baan, J., NOV 1995. Magnetic-Resonance-Imaging Analysis Of Left-ventricular Pressure-Volume Relations - Validation With The Conductance Method At Rest And During Dobutamine Stress. Magn. Reson. Med. $34(5), 728-737$. 
[32] Pilla, J., Blom, A., Brockman, D., Ferrari, V., Yuan, D., Acker, M., NOV 2003. Passive ventricular constraint to improve left ventricular function and mechanics in an ovine model of heart failure secondary to acute myocardial infarction. J. Thorac. Cardiovasc. Surg. 126 (5), 1467-1476.

[33] Ratcliffe, M., Wallace, A., Salahieh, A., Hong, J., Ruch, S., Hall, T., JAN 2000. Ventricular volume, chamber stiffness, and function after anteroapical aneurysm plication in the sheep. J. Thorac. Cardiovasc. Surg. 119 (1), 115-124.

[34] Schmitt, B., Steendijk, P., Lunze, K., Ovroutski, S., Falkenberg, J., Rahmanzadeh, P., Maarouf, N., Ewert, P., Berger, F., Kuehne, T., 2009. Integrated assessment of diastolic and systolic ventricular function using diagnostic cardiac magnetic resonance catheterization: Validation in pigs and application in a clinical pilot study. J. Am. Coll. Cardiol. Img. 2, 1271-1281.

[35] Segers, P., Steendijk, P., Stergiopulos, N., Westerhof, N., JAN 2001. Predicting systolic and diastolic aortic blood pressure and stroke volume in the intact sheep. J. Biomech. 34 (1), 41-50.

[36] Senzaki, H., Chen, C., Kass, D., 1996. Single-beat estimation of end-systolic pressure-volume relation in humans. Circulation 94, 2497-2506.

[37] Shishido, T., Hayashi, K., Shigemi, K., Sato, T., Sugimachi, M., Sunagawa, K., 2000. Single-beat estimation of end-systolic elastance using bilinearly approximated time-varying elastance curve. Circulation 102, 1983-2989.

[38] Spratt, J., Tyson, G., Glower, D., Davis, J., Muhlbaier, L., Olsen, C., Rankin, J., 1987. The end-systolic pressure-volume relationship in conscious dogs. Circulation 75, 1295-1309.

[39] Starling, M., 1993. Left ventricular-arterial coupling relations in the normal human heart. Am. Heart J. $125,1659-1666$.

[40] Suga, H., May 2003. Global cardiac function: mechano-energetico-informatics. J. Biomech 36 (5), 713-720.

[41] Suga, H., Sagawa, K., Shoukas, A., 1973. Load independence of the instantaneous pressure-volume 
ratio of the canine left ventricle and effects of epinephrine and heart rate on the ratio. Circ. Res. 32, 314-322.

[42] Sunagawa, K., Maughan, W., Sagawa, K., 1985. Optimal arterial resistance for the maximal stroke work studied in isolated canine left ventricle. Circ. Res. 56, 586-595.

[43] Sunagawa, K., Yamada, A., Senda, Y., Kikuchi, Y., Nakamura, M. Shibahara, T., Nose, Y., 1980. Estimation of the hydromotive source pressure from ejecting beats of the left ventricle. IEEE Trans Biomed Eng. 27, 299-305.

[44] Takeuchi, M., Igarashi, Y., Tomimoto, S., Odake, M., Hayashi, T., Tsukamoto, T., Hata, K., Takaoka, H., Fukuzaki, H., 1991. Single-beat estimation of the slope of the end-systolic pressure-volume relation in the human left ventricle. Circulation 83, 202-212.

[45] Thomas, J., Weyman, A., 1991. Echocardiographic doppler evaluation of left ventricular diastolic function. physics and physiology. Circulation 84, 977-990.

[46] Varma, S., Owen, R., Smucker, M., Feldman, M., 1989. Is tau a preload-independent measure of isovolumetric relaxation? Circulation $80,1757-1765$.

[47] Weiss, J., Frederisken, J., Weisfeldt, M., 1976. Hemodynamic determinants of the time-course of fall in canine left ventricular pressure. J. Clin. Invest. 58, 751-760.

[48] Winter, E. M., Grauss, R. W., Atsma, D. E., Hogers, B., Poelmann, R. E., van der Geest, R. J., Tschope, C., Schalij, M. J., Groot, A. C. G.-d., Steendijk, P., OCT 2008. Left ventricular function in the post-infarct failing mouse heart by magnetic resonance imaging and conductance catheter: a comparative analysis. Acta Physiol. 194 (2), 111-122.

[49] Zile, M., Brutsaert, D., 2002. New concepts in diastolic dysfunction and diastolic heart failure: Part I:diagnosis, prognosis, and measurements of diastolic function. Circulation 105, 1387-1393. 